\title{
SEM-FIB Characterization of Reverse Osmosis Membrane Fouling
}

\author{
A. R. Behzad*, K. Hooghan ${ }^{* *}$, C. Aubry ${ }^{*}$, M. T. Khan ${ }^{*}$ and J. P. Croue* \\ *King Abdullah University of Science \& Technology (KAUST), Thuwal, 23955, Kingdom of Saudi Arabia \\ (KSA),
}

\section{${ }^{* *}$ FEI Company, 5350 NE Dawson Creek Dr., Hillsboro, OR, 97124}

Membrane fouling is a serious problem for efficient operation of reverse osmosis (RO) water desalination. Precipitated salts, organic materials and micro organisms gradually accumulate on the surface of RO membrane leading to formation of cake layer (fouling) and reduced membrane performance [1]. Surface and cross section analysis of RO membranes are routinely used to investigate the membrane fouling and failure. However, many methods currently used to prepare membranes for surface and cross section analyses suffer from the loss of materials from the cake layer during fixation process as well as artifacts and deformation of cake layer at cut surface [2]. In this study we used cryo fixation, freeze drying and focus ion beam (FIB) to prepare membranes for surface and cross section characterizations.

Fouled RO membranes from pilot scale modules fed with urban waste water were used for this study. A Nova Nano SEM (FEI Company) equipped with cryo preparation chamber (Quorum Technology) was used to analyze surface of cryo-fixed membranes in natural hydrated state. A Quanta 3D) equipped with an electron and focus ion beam (FIB) and cryo-preparation chamber was also used to prepare clean cross sections of cryo-fixed fouled membranes free of artifacts and to examine the cross sectioned plane of the cake layer at low temperature. Freeze drying of membranes was carried out inside a K775X turbo freeze dryer (Quorum technology) and Quanta 3D SEM was used to prepare clean cross sections of these freeze dried membranes. Subsequently, the Nova Nano SEM equipped with EDS detector (EDAX) was used to image the surface and cross sections of freeze dried membranes and to identify the elemental compositions and distribution of inorganic particles present on the surface of cake layer.

Cryo-SEM analysis showed the presence of different types of micro-organisms and inorganic particles at the surface of the cake layer (Fig 1A). FIB sectioning and imaging of cryo-fixed membrane in cross section plane showed internal structure of the cake layer including the fouling materials and embedded microorganisms in cake layer in the natural hydrated state and free of artifacts (Fig. 1B). Alternatively, similar ultrastructural details were observed at the surface of cake layer when freeze dried membrane was examined in SEM (Fig. 2A). Subsequent FIB cross sectioning of freeze dried membrane allowed us to observe the internal structure of cake layer including colloidal particles, microorganisms and their spatial relationship with the membrane (Fig. 2B). The EDS spot and map analyses of freeze dried membranes showed that the inorganic particles present in the cake layer were made of $\mathrm{Fe}, \mathrm{Cr}, \mathrm{Co}, \mathrm{Cu}$ and $\mathrm{Ni}$ while microorganisms and other organic materials contained $\mathrm{Ca}, \mathrm{Si}, \mathrm{O}$ and $\mathrm{K}$ (data no shown). 
In summary, cryo and freeze-drying techniques are quite promising for ultrastructural characterization of fouling. Application of FIB provides artifact free cross sections with sharp ultrastructural details that can help identify the cause of fouling.

\section{References}

1] K.L. Chen et al., J Membr. Sci. 232 (2004) 63-72.

2] R. R. ferlia et al., Env. Progress 27 (2008) 204-209.
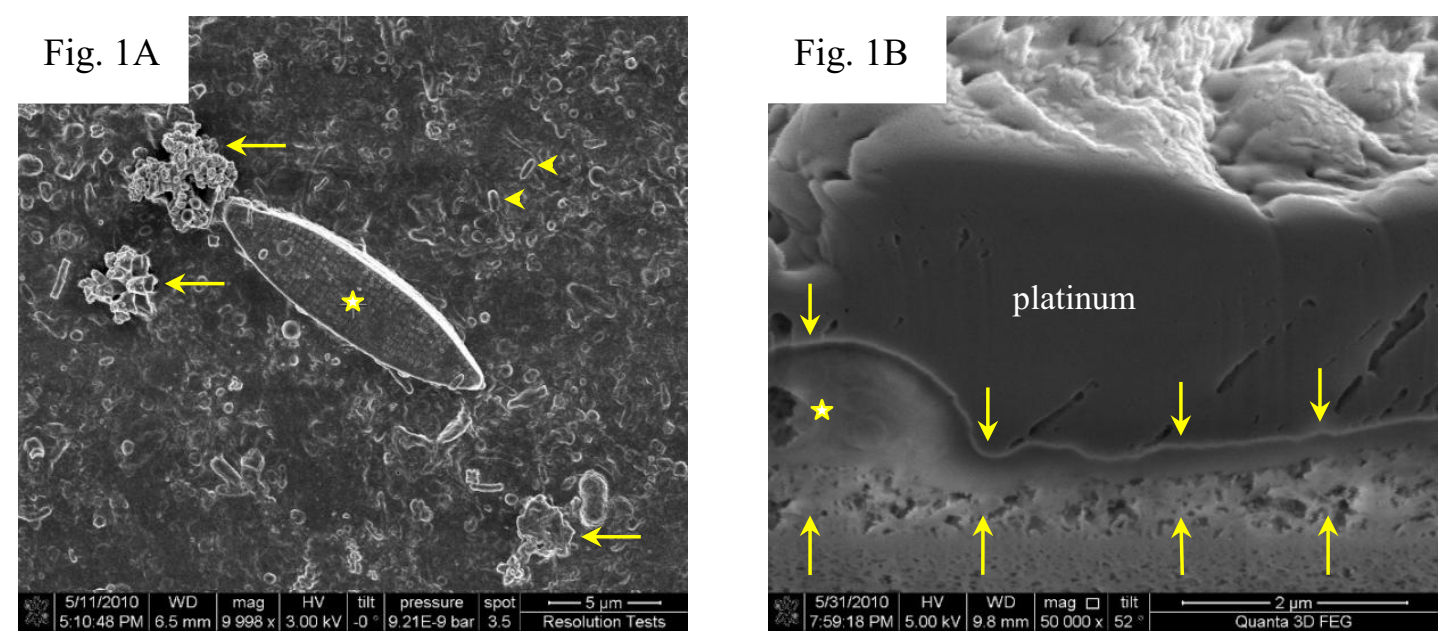

Fig. 1 Cryo-SEM of surface and cross section of fouled membrane. A) diatom (star), rod shape bacteria (arrowheads) and colloidal particles (arrows) are visible on the surface of membrane. B) FIB assisted sectioning of cryo fixed membrane showing the cake layer (between arrows) and the fungal hyphae (star) in cross section.
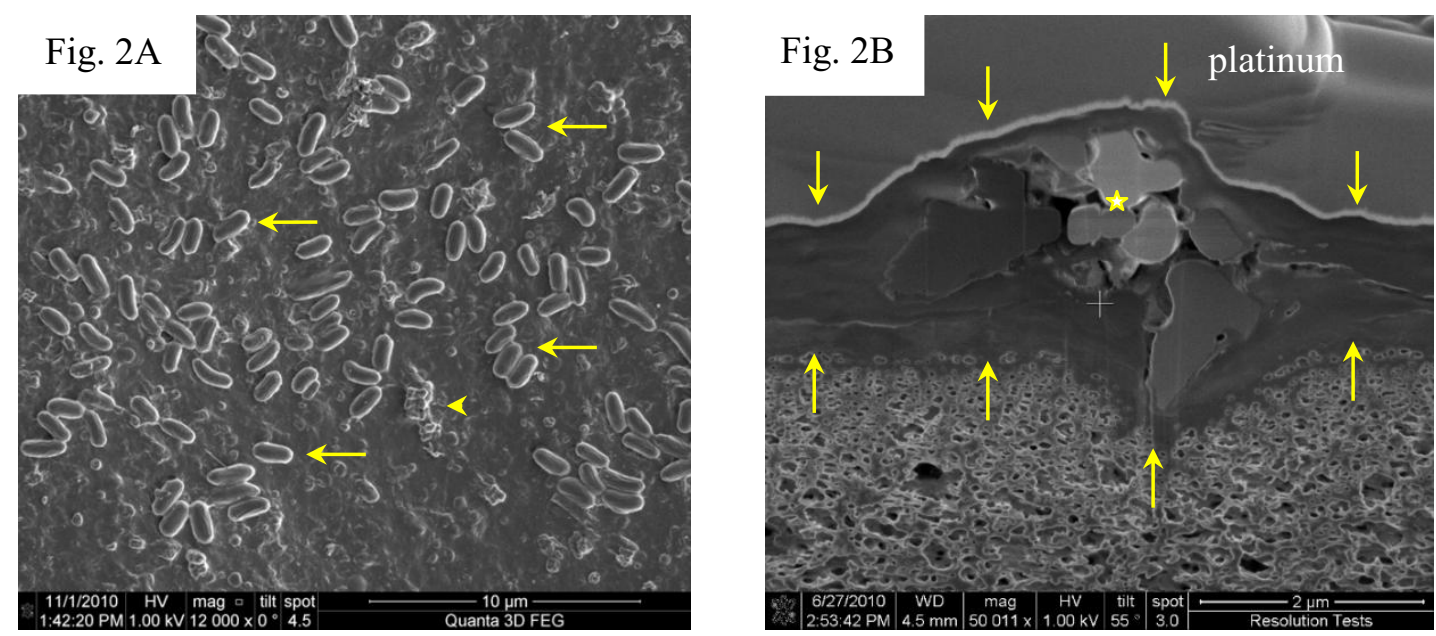

Fig. 2 SEM images of surface and cross section of a freeze dried fouled membrane. A) Rod shape bacteria (arrows) and colloidal particles (arrowhead) on the surface. B) Cross section profile of membrane prepared by FIB showing the cake layer (between arrows) with embedded inorganic particles (star). 